\title{
ON RIESZ SUMMABILITY OF FOURIER SERIES
}

FU CHENG HSIANG

1. Let $\phi(t)$ be an even function, integrable in Lebesgue sense and periodic with period $2 \pi$. Let

$$
\phi(t) \sim \frac{1}{2} a_{0}+\sum_{n=1}^{\infty} a_{n} \cos n t .
$$

Let $\lambda(x)$ be a continuous function, monotone increasing in $(0, \infty)$ and tends to infinity as $x \rightarrow \infty$. The series $\sum a_{n}$ is said to be summable $(R, \lambda(\omega), r)$ if $c_{r}(\omega) /(\lambda(\omega))^{r} \rightarrow s$ as $\omega \rightarrow \infty$, where

$$
c_{r}(\omega)=\frac{1}{2}(\lambda(\omega)-\lambda(0))^{r} a_{0}+\sum_{n \leqq \omega}(\lambda(\omega)-\lambda(n))^{r} a_{n} .
$$

Throughout this note, we assume $s=0$, and on writing $\lambda(x)=e^{\mu(x)}$, $\mu(x)$ satisfies the following conditions (A):

(i) $\mu(x)$ is differentiable and monotone increasing for $x>A$ and $\mu(x)>1$ as $x \rightarrow \infty$,

(ii) $\mu^{\prime}(x)$ is monotone decreasing for $x>A$ and $\mu^{\prime}(x) \rightarrow 0$ as $x \rightarrow \infty$,

(iii) $\left(\mu^{\prime}(x)\right)^{2} \geqq-2 \mu^{\prime \prime}(x) \geqq 0,\left(\mu^{\prime}(x)\right)^{3} \geqq-\left(3 \mu^{\prime}(x) \mu^{\prime \prime}(x)+\mu^{\prime \prime \prime}(x)\right)$ for $x>A$,

$$
U(x)=\int_{x}^{\infty} \frac{d u}{u \mu(u)} \text { exists for } x>A
$$

and

$$
x\left(\mu^{\prime}(x)\right)^{\Delta} U\left(\frac{1}{\mu^{\prime}(x)}\right)=O(1)
$$

for some $0<\Delta<2$,

(b) $x^{\Delta} U(x)$ being monotone increasing for some $0<\Lambda<2-\Delta$, $\mu(x)$ may be taken in the following forms:

(1) $(\log x)^{1+\Delta}(\Delta>0, x>A>1)$, (2) $x^{\alpha}(0<\alpha<1 / 2, x>A>0)$,

$$
\text { (3) } x^{\alpha}(\log x)^{-\Delta} \quad(\Delta>0,0<\alpha<1 / 2, x>A>1) \text {. }
$$

Hardy and Littlewood have derived a convergence test for the oscillating series $\sum a_{n}[2 ; 3 ; 4]$ which contains two conditions: one is

Received by the editors April 8, 1957 and, in revised form, June 21, 1957. 
concerning the behavior of $\phi(t)$ near the point $t=0$, e.g., the continuity condition. The other is concerning the magnitude of $a_{n}$, e.g., the coefficients condition. Morgan [8] developed Hardy-Littlewood's result so as to permit varying types of conditions on $\phi(t)$ and $a_{n}$. Wang $[5 ; 10 ; 11]$ proved Hardy-Littlewood's test in one-sided Tauberian condition for $a_{n}$. Sunouchi [9] joined the results of Morgan and Wang and established another type of convergence test for the series $\sum a_{n}$. Jurkat [6] developed Sunouchi's result as same as Hardy-Littlewood's to further steps. Very recently, Kanno [7] has extended Wang's theorem to the fractional integral $\phi_{\alpha}(t)(\alpha \geqq 1)$ of $\phi(t)$. In the present note, we give the above investigations an exhaustive solution. Our result includes, however, to some degrees, the works of the previous authors. It is known that [10], for $r>0, \omega>0$,

$$
c_{r}(\omega)=\frac{2}{\pi} \int_{0}^{\pi} \phi(t) E_{r}(\omega, t) d t+o\left((\lambda(\omega))^{r}\right),
$$

where

$$
E_{r}(\omega, t)=\frac{r}{t} \int_{0}^{\omega}(\lambda(\omega)-\lambda(x))^{r-1} \lambda^{\prime}(x) \sin x t d x .
$$

In addition, we define:

$$
\lambda(x)=\left\{\begin{array}{lr}
e^{\mu(x)} & (x>A), \\
\lambda(A)+\lambda^{\prime}(A)(x-A) & (0 \leqq x \leqq A) .
\end{array}\right.
$$

2. The following lemmas are required.

Lemma 1. For $\omega>A$,

$$
E_{2}(\omega, t)=O\left(e^{2 \mu(\omega)} \omega\right)
$$

uniformly in $t$,

$$
\begin{aligned}
E_{2}(\omega, t) & =O\left(e^{\mu(\omega)} t^{-2}\right)+O\left(e^{2 \mu(\omega)} \mu^{\prime}(\omega) t^{-2}\right), \\
\frac{\partial}{\partial t} E_{2}(\omega, t) & =O\left(e^{2 \mu(\omega)} \omega t^{-1}\right)
\end{aligned}
$$

and

$$
\frac{\partial}{\partial t} E_{2}(\omega, t)=O\left(e^{\mu(\omega)} t^{-3}\right)+O\left(e^{2 \mu(\omega)}\left(\mu^{\prime}(\omega)\right)^{2} \omega t^{-3}\right) .
$$

Proof. By the second mean value theorem, ${ }^{1}$

${ }^{1} B$ is a numerical constant not necessarily the same at each occurrence. 


$$
\begin{aligned}
E_{2}(\omega, t) & =\int_{0}^{\omega}(\lambda(\omega)-\lambda(x))^{2} \cos x t d x \\
& =(\lambda(\omega)-B)^{2} \int_{0}^{\omega^{\prime}} \cos x t d x \\
& =O\left(e^{2 \mu(\omega)} \omega\right) . \\
\frac{\partial}{\partial t} E_{2}(\omega, t) & =-\int_{0}^{\omega}(\lambda(\omega)-\lambda(x))^{2} x \sin x t d x \\
& =-(\lambda(\omega)-B)^{2} \omega \int_{\omega^{\prime}}^{\omega^{\prime \prime}} \sin x t d x \\
& =O\left(e^{2 \mu(\omega)} \omega t^{-1}\right)
\end{aligned}
$$

for $\omega>A$, by the second mean value theorem, where $0<\omega^{\prime}<\omega^{\prime \prime}<\omega$. Now,

$$
\begin{aligned}
E_{2}(\omega, t) & =\frac{2}{t}\left(\int_{0}^{A}+\int_{A}^{\omega}\right)(\lambda(\omega)-\lambda(x)) \lambda^{\prime}(x) \sin x t d x \\
& =L_{1}+L_{2} . \\
L_{1} & =\frac{2}{t}(\lambda(\omega)-B) \lambda^{\prime}(A) \int_{0}^{\tau} \sin x t d x=O\left(e^{\mu(\omega)} t^{-2}\right), \\
L_{2} & =\frac{2}{t}(\lambda(\omega)-B) \lambda^{\prime}(\omega) \int_{\omega^{\prime}}^{\omega^{\prime \prime}} \sin x t d x,
\end{aligned}
$$

since, by (iii) of the conditions (A), $\lambda^{\prime}(x)$ is monotone increasing in $(A, \omega)$. It follows that

$$
L_{2}=O\left(e^{2 \mu(\omega)} \mu^{\prime}(\omega) t^{-2}\right)
$$

Thus,

$$
E_{2}(\omega, t)=O\left(e^{\mu(\omega)} t^{-2}\right)+O\left(e^{2 \mu(\omega)} \mu^{\prime}(\omega) t^{-2}\right)
$$

for $\omega>A$. Further,

$$
\begin{aligned}
\frac{\partial L_{1}}{\partial t}= & -\frac{2 \lambda^{\prime}(A)}{t^{2}} \int_{0}^{A}(\lambda(\omega)-\lambda(x)) \sin x t d x \\
& +\frac{2 \lambda^{\prime}(A)}{t} \int_{0}^{A}(\lambda(\omega)-\lambda(x)) x \cos x t d x \\
= & O\left(e^{\mu(\omega)} t^{-3}\right) .
\end{aligned}
$$

By integration by parts once, we find 


$$
\begin{aligned}
L_{2}= & \frac{2}{t^{2}}(\lambda(\omega)-\lambda(A)) \lambda^{\prime}(A) \cos A t \\
& +\frac{2}{t^{2}} \int_{A}^{\omega} \frac{d}{d x}\left((\lambda(\omega)-\lambda(x)) \lambda^{\prime}(x) \cos x t d x\right. \\
= & \frac{2}{t^{2}}(\lambda(\omega)-\lambda(A)) \lambda^{\prime}(A) \cos A t \\
& -\frac{2}{t^{2}} \int_{A}^{\omega}\left(\left(\lambda^{\prime}(x)\right)^{2}-(\lambda(\omega)-\lambda(x)) \lambda^{\prime \prime}(x)\right) \cos x t d x .
\end{aligned}
$$

Denote $H(\omega, x)=\left(\lambda^{\prime}(x)\right)^{2}-(\lambda(\omega)-\lambda(x)) \lambda^{\prime \prime}(x)$. Then

$$
\begin{aligned}
\frac{\partial L_{2}}{\partial t}= & \frac{4}{t^{3}}(\lambda(\omega)-\lambda(A)) \lambda^{\prime}(A) \cos A t+\frac{2 A}{t^{2}}(\lambda(\omega)-\lambda(A)) \sin A t \\
& +\frac{4}{t^{3}} \int_{A}^{\omega} H(\omega, x) \cos x t d x+\frac{2}{t^{2}} \int_{A}^{\omega} H(\omega, x) x \sin x t d x \\
= & O\left(e^{\mu(\omega)} t^{-3}\right)+\left(I_{1}+I_{2}+I_{3}+I_{4}\right) . \\
I_{1}= & \frac{4}{t^{3}} \int_{A}^{\omega}\left(\lambda^{\prime}(x)\right)^{2} \cos x t d x=\frac{4}{t^{3}}\left(\lambda^{\prime}(\omega)\right)^{2} \int_{\omega^{\prime}}^{\omega} \cos x t d x \\
= & O\left(\left(\lambda^{\prime}(\omega)\right)^{2} \omega t^{-3}\right) . \\
I_{2}= & -\frac{4}{t^{3}} \int_{A}^{\omega}(\lambda(\omega)-\lambda(x)) \lambda^{\prime \prime}(x) \cos x t d x \\
= & -\frac{4}{t^{3}}(\lambda(\omega)-B) \lambda^{\prime \prime}(\omega) \int_{\omega^{\prime}}^{\omega^{\prime \prime}} \cos x t d x
\end{aligned}
$$

since, by (iii) of the conditions $(A), \lambda^{\prime \prime}(x)$ is monotone increasing in $(A, \omega)$. It follows that

$$
\begin{aligned}
I_{2} & =O\left(t^{-3}(\lambda(\omega)-B) e^{\mu(\omega)}\left(\left(\mu^{\prime}(\omega)\right)^{2}+\mu^{\prime \prime}(\omega)\right) \omega\right) \\
& =O\left(e^{2 \mu(\omega)}\left(\mu^{\prime}(\omega)\right)^{2} \omega t^{-3}\right)+O\left(e^{2 \mu(\omega)}\left|\mu^{\prime \prime}(\omega)\right| \omega t^{-3}\right) .
\end{aligned}
$$

In view of $\left(\mu^{\prime}(x)\right)^{2} \geqq-2 \mu^{\prime \prime}(x) \geqq 0$ for $x>A$, the second term can be absorbed in the first, so that

$$
\begin{aligned}
I_{2} & =O\left(e^{2 \mu(\omega)}\left(\mu^{\prime}(\omega)\right)^{2} \omega t^{-3}\right) . \\
I_{2} & =\frac{2}{t^{2}} \int_{A}^{\omega}\left(\lambda^{\prime}(x)\right)^{2} x \sin x t d x \\
& =\frac{2}{t^{2}}\left(\lambda^{\prime}(\omega)\right)^{2} \omega \int_{\omega^{\prime}}^{\omega} \sin x t d x=O\left(\left(\lambda^{\prime}(\omega)\right)^{2} \omega t^{-3}\right) .
\end{aligned}
$$




$$
\begin{aligned}
I_{4} & =-\frac{2}{t^{2}} \int_{A}^{\omega}(\lambda(\omega)-\lambda(x)) \lambda^{\prime \prime}(x) x \sin x t d x \\
& =-\frac{2}{t^{2}}(\lambda(\omega)-B) \lambda^{\prime \prime}(\omega) \omega \int_{\omega^{\prime}}^{\omega^{\prime \prime}} \sin x t d x \\
& =-\frac{2}{t^{2}}(\lambda(\omega)-B) e^{\mu(\omega)}\left(\left(\mu^{\prime}(\omega)\right)^{2}+\mu^{\prime \prime}(\omega)\right) \omega \int_{\omega^{\prime}}^{\omega^{\prime \prime}} \sin x t d x \\
& =O\left(e^{2 \mu(\omega)}\left(\mu^{\prime}(\omega)\right)^{2} \omega t^{-3}\right)+O\left(e^{2 \mu(\omega)}\left|\mu^{\prime \prime}(\omega)\right| \omega t^{-3}\right) \\
& =O\left(e^{2 \mu(\omega)}\left(\mu^{\prime}(\omega)\right)^{2} \omega t^{-3}\right) .
\end{aligned}
$$

Thus, $(\partial / \partial t) E_{2}(\omega, t)=O\left(e^{\mu(\omega)} t^{-3}\right)+O\left(e^{2 \mu(\omega)}\left(\mu^{\prime}(\omega)\right)^{2} \omega t^{-3}\right)$ for $\omega>A$. This proves the lemma.

LEMmA 2. If $\sum a_{n}$ is summable $(R, \lambda(\omega), r)$ and

$$
a_{n}>-K \frac{\lambda(n)-\lambda(n-1)}{\lambda(n)}
$$

then $\sum a_{n}$ converges.

This lemma is known. [1].

3. Now, we are in a position to establish the following

THEOREM 1. If

$$
\phi_{1}(t)=\int_{0}^{t} \phi(u) d u=o\left(t^{\Delta} U\left(\frac{1}{t}\right)\right) \quad(t \rightarrow+0),
$$

then the series $\sum a_{n}$ is summable $(R, \lambda(\omega), 2)$ to zero.

Proof. We have

$$
\begin{aligned}
\frac{\pi}{2} c_{2}(\omega) & =\int_{0}^{\pi} \phi(t) E_{2}(\omega, t) d t+o\left(e^{2 \mu(\omega)}\right) \\
& =\left(\int_{0}^{1 / A-\epsilon}+\int_{1 / A-\epsilon}^{\pi}\right) \phi(t) E_{2}(\omega, t) d t+o\left(e^{2 \mu(\omega)}\right) .
\end{aligned}
$$

By the second relation of Lemma 1 ,

$$
\begin{aligned}
& \int_{1 / A-\epsilon}^{\pi} \phi(t) E_{2}(\omega, t) d t \\
& =O\left(e^{\mu(\omega)} \int_{1 / A-\epsilon}^{\pi} \frac{|\phi(t)|}{t^{2}} d t\right)+O\left(e^{2 \mu(\omega)} \mu^{\prime}(\omega) \int_{1 / A-\epsilon}^{\pi} \frac{|\phi(t)|}{t^{2}} d t\right) \\
& =O\left(e^{\mu(\omega)}\right)+O\left(e^{2 \mu(\omega)} \mu^{\prime}(\omega)\right)=o\left(e^{2 \mu(\omega)}\right),
\end{aligned}
$$


since $\mu^{\prime}(\omega) \rightarrow 0$ as $\omega \rightarrow \infty$. By integration by parts,

$$
\int_{0}^{1 / A-\epsilon} \phi(t) E_{2}(\omega, t) d t=\left(\phi_{1}(t) E_{2}(\omega, t)\right)_{0}^{1 / A-\epsilon}-\int_{0}^{1 / A-\epsilon} \phi_{1}(t) \frac{\partial}{\partial t} E_{2}(\omega, t) d t .
$$

The integrated term is easily seen to be $o\left(e^{2 \mu(\omega)}\right)$ by the first and the second relations of Lemma 1 . Write

$$
\begin{aligned}
\int_{0}^{1 / A-\epsilon} \phi_{1}(t) \frac{\partial}{\partial t} E_{2}(\omega, t) d t & =\left(\int_{0}^{\mu^{\prime}(\omega)}+\int_{\mu^{\prime}(\omega)}^{1 / A-\epsilon}\right) \phi_{1}(t) \frac{\partial}{\partial t} E_{2}(\omega, t) d t \\
& =J_{1}+J_{2} . \\
J_{1} & =o\left(e^{2 \mu(\omega)} \omega \int_{0}^{\mu^{\prime}(\omega)} t^{\Delta-1} U\left(\frac{1}{t}\right) d t\right) \\
& =o\left(e^{2 \mu(\omega)} \omega\left(\mu^{\prime}(\omega)\right)^{\Delta} U\left(\frac{1}{\mu^{\prime}(\omega)}\right)\right) \\
& =o\left(e^{2 \mu(\omega)}\right)
\end{aligned}
$$

by (iv) of the conditions $(A)$.

$$
\begin{aligned}
J_{2}= & \int_{\mu^{\prime}(\omega)}^{1 / A-\epsilon} \phi_{1}(t) \frac{\partial}{\partial t} E_{2}(\omega, t) d t \\
= & o\left(e^{\mu(\omega)} \int_{\mu^{\prime}(\omega)}^{1 / A-\epsilon} t^{\Delta-3} U(t) d t\right)+o\left(e^{2 \mu(\omega)}\left(\mu^{\prime}(\omega)\right)^{2} \omega \int_{\mu^{\prime}(\omega)}^{1 / A-\epsilon} t^{\Delta-3} U(t) d t\right) \\
= & o\left(e^{\mu(\omega)}\left(\mu^{\prime}(\omega)\right)^{-\Lambda} U\left(\frac{1}{\mu^{\prime}(\omega)}\right) \int_{\mu^{\prime}(\omega)}^{\tau} t^{\Delta+\Lambda-3} d t\right) \\
& +o\left(e^{2 \mu(\omega)}\left(\mu^{\prime}(\omega)\right)^{2-\Lambda} \omega U\left(\frac{1}{\mu^{\prime}(\omega)}\right) \int_{\mu^{\prime}(\omega)}^{\tau} t^{\Delta+\Lambda-3} d t\right) \\
= & o\left(e^{\mu(\omega)}\left(\mu^{\prime}(\omega)\right)^{\Delta-2} U\left(\frac{1}{\mu^{\prime}(\omega)}\right)\right)+o\left(e^{2 \mu(\omega)}\left(\mu^{\prime}(\omega)\right)^{\Delta} \omega U\left(\frac{1}{\mu^{\prime}(\omega)}\right)\right) \\
= & o\left(e^{2 \mu(\omega)}\left(\mu(\omega)\left(\mu^{\prime}(\omega)\right)^{2}\right)^{-1}\left(\mu^{\prime}(\omega)\right)^{\Delta} U\left(\frac{1}{\mu^{\prime}(\omega)}\right)\right)+o\left(e^{2 \mu(\omega)}\right) \\
= & o\left(e^{2 \mu(\omega)}\right)
\end{aligned}
$$

since $e^{\mu(x)}\left(\mu^{\prime}(x)\right)^{2}$ is positive and monotone increasing for $x>A$ by (iii) of the conditions $(A)$. This proves the theorem.

By means of Theorem 1 and Lemma 2, we obtain immediately

TheOREM 2. If

$$
\phi_{1}(t)=o\left(t^{\Delta} U\left(\frac{1}{t}\right)\right) \quad(t \rightarrow+0),
$$




$$
a_{n}>-K \frac{\lambda(n)-\lambda(n-1)}{\lambda(n)}
$$

for some $K>0$, then $\sum a_{n}$ converges to zero.

4. Last, we illustrate by some special cases derived from our result:

(a) Let $0<\alpha<1 / 2$. If

$$
\begin{aligned}
\phi_{1}(t) & =o\left(t^{1 /(1-\alpha)}\left(\log \frac{1}{t}\right)^{\Delta}\right) \quad(t \rightarrow+0), \\
a_{n} & >-K n^{\alpha-1}(\log n)^{-\Delta}
\end{aligned}
$$

for some $K>0$, then $\sum a_{n}$ converges to zero.

(b) Let $0<\alpha<1 / 2$. If

$$
\begin{gathered}
\phi_{1}(t)=o\left(t^{1 /(1-\alpha)}\right) \\
a_{n}>-K n^{\alpha-1}
\end{gathered}
$$$$
(t \rightarrow+0)
$$

then $\sum a_{n}$ converges to zero.

(c) If

$$
\begin{gathered}
\phi_{1}(t)=o\left(t\left(\log \frac{1}{t}\right)^{-a}\right) \\
a_{n}>K n^{-1}(\log n)^{\Delta}
\end{gathered}
$$

for some $\Delta>0$, then $\sum a_{n}$ converges to zero.

\section{REFERENCES}

1. G. H. Hardy, An extension of a theorem on the oscillating series, Proc. London Math. Soc. vol. 12 (1913) pp. 174-180.

2. G. H. Hardy and J. E. Littlewood, Notes on the theory of series (XVII): Some new convergence criteria for Fourier series, J. London Math. Soc. vol. 7 (1932) pp. 252256.

3. - Some new convergence criteria for Fourier series, Annali della Scuola Normale Superiore di Pisa vol. 3 (1934) pp. 1-20.

4. G. H. Hardy and W. W. Rogosinski, Fourier series, Cambridge, 1946, pp. 45, 96.

5. F. C. Hsiang, On a test for the convergence of Fourier series, Proc. Amer. Math. Soc. vol. 7 (1956) pp. 1036-1039.

6. W. Jurkat, Zur Convergenztheorie der Fourier-Riehen, Math. Zeit. vol. 53 (1950) pp. 309-339.

7. K. Kanno, On the Riesz summability of Fourier series, Tôhoku Math. J. vol. 8 (1956) pp. 223-234.

8. W. G. Morgan, On the convergence criteria for Fourier series of Hardy and Littlewood, Annali della Scuola Normale Superiore di Pisa vol. 4 (1935) pp. 337-382.

9. G. Sunouchi, Notes on Fourier series: I, On the convergence test of Fourier series, Mathematica Japanica vol. 1 (1948-49) pp. 41-44. 
10. F. T. Wang, On Riesz summability of Fourier series, Proc. London Math. Soc. (2) vol. 47 (1942) pp. 308-325.

11. - On Riesz summability of Fourier series (III), Proc. London Math. Soc. (2) vol. 51 (1949) pp. 215-231.

National TaIwan University

\title{
ON THE IDENTITY OF FUNCTION SPACES ON CARTESIAN PRODUCT SPACES ${ }^{1}$
}

\author{
JOHN C. HOLLADAY
}

For $i=1, \cdots, n$, let $S_{i}$ be a compact Hausdorff space and $F_{i}$ be a closed linear subspace of the complex Banach space $C\left(S_{i}\right)$, the set of all continuous functions from $S_{i}$ to the complex numbers. Let $S_{1} \times \cdots \times S_{n}$ be the Cartesian Product of $S_{1}, \cdots, S_{n}$.

Define $F_{1} * \cdots * F_{n}$ as $\left\{\phi \mid \phi \in C\left(S_{1} \times \cdots \times S_{n}\right)\right.$; for any $i$ $=1, \cdots, n$ and $\left(s_{1}, \cdots, s_{i-1}, s_{i+1}, \cdots, s_{n}\right), \phi\left(s_{1}, \cdots, s_{i-1}, \cdot\right.$, $\left.\left.s_{i+1}, \cdots, s_{n}\right) \in F_{i}\right\}$. Also, define $F_{1} \otimes \cdots \otimes F_{n}$ as the closure of the space of linear combinations of functions of the form $\phi\left(s_{1}, \cdots, s_{n}\right)$ $=f_{1}\left(s_{1}\right) \times \cdots \times f_{n}\left(s_{n}\right)$ where each $f_{i} \in F_{i}$, where we base the topology on the norm, $\|\phi\|=\max _{s_{1}}, \ldots, s_{n}\left|\phi\left(s_{1}, \cdots, s_{n}\right)\right|$.

It is easily shown that $F_{1} \otimes \cdots \otimes F_{n}$ is a subspace of $F_{1} * \cdots * F_{n}$ and that if $F_{2}$ is one-dimensional, then $F_{1} \otimes F_{2}=F_{1} * F_{2}$. Furthermore, by using continuous partitions of unity, one may show that $F_{1} \otimes C\left(S_{2}\right)$ $=F_{1} * C\left(S_{2}\right)$. Therefore, if all but at most one of the $F_{i}$ are either onedimensional or $C\left(S_{i}\right)$, then $F_{1} \otimes \cdots \otimes F_{n}=F_{1} * \cdots * F_{n}$. However, it is not known whether for all cases $F_{1} \otimes \cdots \otimes F_{n}$ will equal $F_{1} * \cdots * F_{n}$ or not. ${ }^{2}$ Although this question is not fully answered here, the purpose of this paper is to give a partial answer to this question. The results and arguments of this paper also apply to realvalued function spaces.

1. Lemma. Let $F$ be a closed linear subspace of $C\left(S_{1}\right)$ and $G$ a closed linear subspace of $C\left(S_{2}\right)$. Let $H$ be a closed subspace of $G$ differing from $G$ by only one dimension. Then $F \otimes G=F * G$ implies that $F \otimes H=F * H$.

Received by the editors October 22, 1956 and, in revised form, April 16, 1957.

1 A small part of the work done under an AEC Predoctoral Fellowship at Yale University, year 1952-1953, under the kind and patient guidance of Dr. Charles E. Rickart.

2 Under Proposition 37 of his first paper of the Amer. Math. Soc. Memoirs, no. 16, Alexandre Grothendieck discusses a number of conjectures which are equivalent to this one. 